\section{Basilar Artery}

Elliot J. Roth

Department of Physical Medicine and

Rehabilitation, Northwestern University, Feinberg School of Medicine, Chicago, IL, USA

\section{Definition}

The basilar artery provides blood to the brain. This artery and the two vertebral arteries comprise the vertebrobasilar system, which supplies blood to the posterior part of circle of Willis and connects ("anastomoses") with blood supplied to the anterior part of the circle of Willis from the carotid arteries. It arises from the confluence of the two vertebral arteries, next to the lower brain stem, ascends parallel to the brain stem, and gives rise to the anterior inferior cerebellar artery, which supplies part of the cerebellum, some smaller branches that supply the brain stem, and the superior cerebellar artery. It finally divides into the two posterior cerebral arteries (PCA). These supply the upper brain stem, the occipital lobe, and the posterior portion of the temporal lobes.

\section{Current Knowledge}

The clinical manifestations of basilar artery occlusion depend on the location of the occlusion, the extent of thrombus, and the collateral flow. Normally, the blood flows in an anterograde fashion from the vertebral arteries to the basilar artery up to its terminal branches. This pattern of flow may vary. If the proximal segment of the basilar artery is occluded and the occlusion resulted from a slowly progressive stenosis, collateralization occurs within the cerebellum into the circumferential branches of the basilar artery. In addition, flow can be reversed from the PCAs into the distal basilar artery. Thrombosis of the basilar artery causes various clinical syndromes that result from brainstem ischemia, including cranial nerve dysfunction, difficulty in swallowing and breathing, and at its most severe, locked-in syndrome. Basilar artery thrombosis is the most common cause of locked-in syndrome. Mortality rate of basilar artery occlusion is $70 \%$, but this can be reduced substantially through the use of antithrombotic agents.

\section{Cross-References}

$\checkmark$ Circle of Willis

- Posterior Cerebral Artery

- Vertebrobasilar System 